\title{
Optical diffraction tomography techniques for the study of cell pathophysiology
}

\author{
Kyoohyun Kim ${ }^{1,2}$, Jonghee Yoon ${ }^{1,2}$, Seungwoo Shin ${ }^{1,2}$, SangYun Lee ${ }^{1,2}$, Su-A Yang ${ }^{3}$, \\ and YongKeun Park ${ }^{1,2,4^{*}}$ \\ ${ }^{1}$ Department of Physics, Korea Advanced Institute of Science and Technology (KAIST), \\ Daejeon 34141, Republic of Korea \\ ${ }^{2}$ KAIST Institute of Health Science and Technology, Daejeon 34141, Republic of Korea \\ ${ }^{3}$ Department of Biological Sciences, KAIST, Daejeon 34141, Republic of Korea \\ ${ }^{4}$ TOMOCUBE, Inc., Daejeon 34051, Republic of Korea \\ *e-mail: yk.park@kaist.ac.kr
}

\begin{abstract}
Three-dimensional imaging of biological cells is crucial for the investigation of cell biology, providing valuable information to reveal the mechanisms behind pathophysiology of cells and tissues. Recent advances in optical diffraction tomography (ODT) have demonstrated the potential for the study of various cells with its unique advantages of quantitative and label-free imaging capability. To provide insight on this rapidly growing field of research and to discuss its applications in biology and medicine, we present the summary of the ODT principle and highlight recent studies utilizing ODT with the emphasis on the applications to the pathophysiology of cells. (C) 2016 Journal of Biomedical Photonics \& Engineering.
\end{abstract}

Keywords: optical diffraction tomography, quantitative phase imaging, biomedical imaging

Paper \#2994 received 2016.03.02; revised manuscript received 2016.05.23; accepted for publication 2016.05.23; published online 2016.06.14. doi: 10.18287/JBPE16.02.020201

\section{References}

1. D. J. Stephens, and V. J. Allan, "Light microscopy techniques for live cell Imaging," Science 300, 82-86 (2003).

2. M. Minsky, "Microscopy apparatus," US 3,013,467 (Dec. 19 1961).

3. W. Denk, J. P. Strickler, and W. W. Webb, "Two-photon laser microscopy," US 5,034,613 (Jul. 23 1991).

4. B. Huang, M. Bates, and X. Zhuang, "Super resolution fluorescence microscopy," Annual Review of Biochemistry 78, 993-1016 (2009).

5. E. Wolf, "Three-dimensional structure determination of semi-transparent objects from holographic data," Optics Communications 1, 153-156 (1969).

6. R. Dändliker, and K. Weiss, "Reconstruction of the three-dimensional refractive index from scattered waves," Optics Communications 1(7), 323-328 (1970).

7. A. F. Fercher, H. Bartelt, H. Becker, and E. Wiltschko, "Image-Formation by Inversion of Scattered Field Data - Experiments and Computational Simulation," Applied Optics 18(14), 2427-2439 (1979).

8. N. Streibl, "Three-dimensional imaging by a microscope," Journal of the Optical Society of America A 2(2), 121-127 (1985).

9. S. Kawata, O. Nakamura, and S. Minami, "Optical Microscope Tomography .1. Support Constraint," Journal of the Optical Society of America A 4(1), 292-297 (1987).

10. T. Noda, S. Kawata, and S. Minami, "Three-dimensional phase contrast imaging by an annular illumination microscope," Applied Optics 29(26), 3810-3815 (1990).

11. A. J. Devaney, and A. Schatzberg, "Coherent optical tomographic microscope," Proc. of SPIE 1767, 62-71 (1992).

12. G. Vishnyakov and G. Levin, "Optical microtomography of phase objects," Optics and Spectroscopy 85(1), 73-77 (1998). 
13. A. C. Kak, and M. Slaney, "Principles of computerized tomographic imaging," Classics in applied mathematics (Society for Industrial and Applied Mathematics, Philadelphia, 2001). ISBN: 978-0-89871-4944.

14. O. Haeberle, K. Belkebir, H. Giovaninni, and A. Sentenac, "Tomographic diffractive microscopy: basics, techniques and perspectives," Journal of Modern Optics 57(9), 686-699 (2010).

15. P. Müller, M. Schürmann, and J. Guck, "The Theory of Diffraction Tomography," arXiv preprint (2015).

16. T. Kim, R. Zhou, L. L. Goddard, and G. Popescu, "Solving inverse scattering problems in biological samples by quantitative phase imaging," Laser \& Photonics Reviews 10, 13-39 (2016).

17. G. Popescu, Quantitative phase imaging of cells and tissues, 1st. ed., McGraw-Hill biophotonics, New York (2011).

18. K. Lee, K. Kim, J. Jung, J. Heo, S. Cho, S. Lee, G. Chang, Y. Jo, H. Park, and Y. Park, “Quantitative Phase Imaging Techniques for the Study of Cell Pathophysiology: From Principles to Applications," Sensors 13(4), 4170-4191 (2013).

19. M. K. Kim, "Principles and techniques of digital holographic microscopy," SPIE Reviews 1(1), 018005 (2010).

20. S. K. Debnath, and Y. Park, "Real-time quantitative phase imaging with a spatial phase-shifting algorithm," Optics Letters 36(23), 4677-4679 (2011).

21. V. Lauer, "New approach to optical diffraction tomography yielding a vector equation of diffraction tomography and a novel tomographic microscope," Journal of Microscopy 205(2), 165-176 (2002).

22. W. Choi, C. Fang-Yen, K. Badizadegan, S. Oh, N. Lue, R. R. Dasari, and M. S. Feld, "Tomographic phase microscopy," Nature Methods 4, 717-719 (2007).

23. Y. Cotte, F. Toy, P. Jourdain, N. Pavillon, D. Boss, P. Magistretti, P. Marquet, and C. Depeursinge, "Markerfree phase nanoscopy," Nature Photonics 7(2), 113-117 (2013).

24. K. Kim, Z. Yaqoob, K. Lee, J. W. Kang, Y. Choi, P. Hosseini, P. T. C. So, and Y. Park, "Diffraction optical tomography using a quantitative phase imaging unit," Optics Letters 39(24), 6935-6938 (2014).

25. A. Barty, K. A. Nugent, A. Roberts, and D. Paganin, "Quantitative phase tomography," Optics Communications 175(4-6), 329-336 (2000).

26. F. Charrière, A. Marian, F. Montfort, J. Kuehn, T. Colomb, E. Cuche, P. Marquet, and C. Depeursinge, "Cell refractive index tomography by digital holographic microscopy," Optics Letters 31(2), 178-180 (2006).

27. A. Kuś, M. Dudek, B. Kemper, M. Kujawińska, and A. Vollmer, "Tomographic phase microscopy of living three-dimensional cell cultures," Journal of Biomedical Optics 19(4), 046009 (2014).

28. A. Kuś, W. Krauze, and M. Kujawińska, "Limited-angle, holographic tomography with optically controlled projection generation," Proc. of SPIE 9330, 933007 (2015).

29. S. Shin, K. Kim, J. Yoon, and Y. Park, "Active illumination using a digital micromirror device for quantitative phase imaging," Optics Letters 40(22), 5407-5410 (2015).

30. S. Shin, K. Kim, T. Kim, J. Yoon, K. Hong, J. Park, and Y. Park, "Optical diffraction tomography using a digital micromirror device for stable measurements of 4-D refractive index tomography of cells," Proc. of SPIE 9718, 971814 (2016).

31. W.-H. Lee, "Binary computer-generated holograms," Applied Optics 18(21), 3661-3669 (1979).

32. W. Gorski, "Tomographic microinterferometry of optical fibers," Optical Engineering 45(12), 125002 (2006).

33. J. Kostencka, T. Kozacki, A. Kus, and M. Kujawinska, "Accurate approach to capillary-supported optical diffraction tomography," Optics Express 23(6), 7908-7923 (2015).

34. P. Memmolo, L. Miccio, F. Merola, O. Gennari, P. A. Netti, and P. Ferraro, "3D morphometry of red blood cells by digital holography," Cytometry part A 85(12), 1030-1036 (2014).

35. M. Habaza, B. Gilboa, Y. Roichman, and N. T. Shaked, "Tomographic phase microscopy with 180 degrees rotation of live cells in suspension by holographic optical tweezers," Optics Letters 40(8), 1881-1884 (2015).

36. P. Müller, M. Schürmann, C. J. Chan, and J. Guck, "Single-cell diffraction tomography with optofluidic rotation about a tilted axis," Proc. of SPIE 9548, 95480U (2015).

37. K. G. Phillips, S. L. Jacques, and O. J. McCarty, "Measurement of single cell refractive index, dry mass, volume, and density using a transillumination microscope," Physical Review Letters 109(11), 118105 (2012).

38. K. Kim, H. Yoon, M. Diez-Silva, M. Dao, R. R. Dasari, and Y. Park, "High-resolution three-dimensional imaging of red blood cells parasitized by Plasmodium falciparum and in situ hemozoin crystals using optical diffraction tomography," Journal of Biomedical Optics 19(1), 011005 (2014).

39. P. Bon, S. Aknoun, S. Monneret, and B. Wattellier, "Enhanced 3D spatial resolution in quantitative phase microscopy using spatially incoherent illumination," Optics Express 22(7), 8654-8671 (2014).

40. L. Yu and M. K. Kim, "Wavelength-scanning digital interference holography for tomographic threedimensional imaging by use of the angular spectrum method," Optics Letters 30(16), 2092-2094 (2005).

41. J. Kühn, F. Montfort, T. Colomb, B. Rappaz, C. Moratal, N. Pavillon, P. Marquet, and C. Depeursinge, "Submicrometer tomography of cells by multiple-wavelength digital holographic microscopy in reflection," Optics Letters 34(5), 653-655 (2009). 
42. S. O. Isikman, W. Bishara, S. Mavandadi, W. Y. Frank, S. Feng, R. Lau, and A. Ozcan, "Lens-free optical tomographic microscope with a large imaging volume on a chip," Proceedings of the National Academy of Sciences of the United States of America 108(18), 7296-7301 (2011).

43. R. Horstmeyer, and C. Yang, "Diffraction tomography with Fourier ptychography," arXiv preprint (2015).

44. W. Gorski and M. Kujawinska, "Three-dimensional reconstruction of refractive index inhomogeneities in optical phase elements," Optics and Lasers in Engineering 38(6), 373-385 (2002).

45. F. Charriere, N. Pavillon, T. Colomb, C. Depeursinge, T. J. Heger, E. A. D. Mitchell, P. Marquet, and B. Rappaz, "Living specimen tomography by digital holographic microscopy: morphometry of testate amoeba," Optics Express 14(16), 7005-7013 (2006).

46. Y. Park, M. Diez-Silva, G. Popescu, G. Lykotrafitis, W. Choi, M. S. Feld, and S. Suresh, "Refractive index maps and membrane dynamics of human red blood cells parasitized by Plasmodium falciparum," Proceedings of the National Academy of Sciences of the United States of America 105(37), 13730-13735 (2008).

47. R. Chandramohanadas, Y. Park, L. Lui, A. Li, D. Quinn, K. Liew, M. Diez-Silva, Y. Sung, M. Dao, C. T. Lim, P. R. Preiser, and S. Suresh, "Biophysics of malarial parasite exit from infected erythrocytes," PloS One 6(6), e20869 (2011).

48. Y. J. Sung, W. Choi, C. Fang-Yen, K. Badizadegan, R. R. Dasari, and M. S. Feld, "Optical diffraction tomography for high resolution live cell imaging," Optics Express 17(1), 266-277 (2009).

49. W. Gorski and W. Osten, "Tomographic imaging of photonic crystal fibers," Optics Letters 32(14), 1977-1979 (2007).

50. T. M. Habashy, R. W. Groom, and B. R. Spies, "Beyond the Born and Rytov Approximations - a Nonlinear Approach to Electromagnetic Scattering," Journal of Geophysical Research-Solid Earth 98(B2), 1759-1775 (1993).

51. A. J. Devaney, "Inverse-Scattering Theory within the Rytov Approximation," Optics Letters 6(8), 374-376 (1981).

52. B. Q. Chen, and J. J. Stamnes, "Validity of diffraction tomography based on the first Born and the first Rytov approximations," Applied Optics 37(14), 2996-3006 (1998).

53. S. S. Kou, and C. J. R. Sheppard, "Image formation in holographic tomography," Optics Letters 33(20), 2362$2364(2008)$.

54. S. S. Kou, and C. J. R. Sheppard, "Image formation in holographic tomography: high-aperture imaging conditions," Applied Optics 48(34), H168-H175 (2009).

55. S. Vertu, J. J. Delaunay, I. Yamada, and O. Haeberle, "Diffraction microtomography with sample rotation: influence of a missing apple core in the recorded frequency space," Central European Journal of Physics 7(1), 22-31 (2009).

56. J. Lim, K. Lee, K. H. Jin, S. Shin, S. Lee, Y. Park, and J. C. Ye, "Comparative study of iterative reconstruction algorithms for missing cone problems in optical diffraction tomography," Optics Express 23(13), 16933-16948 (2015).

57. Y. J. Sung, and R. R. Dasari, "Deterministic regularization of three-dimensional optical diffraction tomography," Journal of the Optical Society of America A 28(8), 1554-1561 (2011).

58. U. S. Kamilov, I. N. Papadopoulos, M. H. Shoreh, A. Goy, C. Vonesch, M. Unser, and D. Psaltis, "Learning approach to optical tomography," Optica 2(6), 517-522 (2015).

59. S. Vertu, J. Flugge, J. J. Delaunay, and O. Haeberle, "Improved and isotropic resolution in tomographic diffractive microscopy combining sample and illumination rotation," Central European Journal of Physics 9(4), 969-974 (2011).

60. R. Barer, "Interference microscopy and mass determination," Nature 169(4296), 366-367 (1952).

61. G. Popescu, Y. Park, N. Lue, C. Best-Popescu, L. Deflores, R. R. Dasari, M. S. Feld, and K. Badizadegan, "Optical imaging of cell mass and growth dynamics," American Journal of Physiology-Cell Physiology 295(2), C538-C544 (2008).

62. K. E. Handwerger, J. A. Cordero, and J. G. Gall, "Cajal bodies, nucleoli, and speckles in the Xenopus oocyte nucleus have a low-density, sponge-like structure," Molecular Biology of the Cell 16(1), 202-211 (2005).

63. M. Mir, Z. Wang, Z. Shen, M. Bednarz, R. Bashir, I. Golding, S. G. Prasanth, and G. Popescu, "Optical measurement of cycle-dependent cell growth," Proceedings of the National Academy of Sciences of the United States of America 108(32), 13124-13129 (2011).

64. H. Y. Zhao, P. H. Brown, and P. Schuckt, "On the Distribution of Protein Refractive Index Increments," Biophysical Journal 100(9), 2309-2317 (2011).

65. Y. J. Sung, A. Tzur, S. Oh, W. Choi, V. Li, R. R. Dasari, Z. Yaqoob, and M. W. Kirschner, "Size homeostasis in adherent cells studied by synthetic phase microscopy," Proceedings of the National Academy of Sciences of the United States of America 110(41), 16687-16692 (2013).

66. Y. Kim, H. Shim, K. Kim, H. Park, S. Jang, and Y. Park, "Profiling individual human red blood cells using common-path diffraction optical tomography," Scientific Reports 4, 6659 (2014). 
67. Y. Kim, H. Shim, K. Kim, H. Park, J. H. Heo, J. Yoon, C. Choi, S. Jang, and Y. Park, "Common-path diffraction optical tomography for investigation of three-dimensional structures and dynamics of biological cells," Optics Express 22(9), 10398-10407 (2014).

68. H. Park, T. Ahn, K. Kim, S. Lee, S. Y. Kook, D. Lee, I. B. Suh, S. Na, and Y. Park, "Three-dimensional refractive index tomograms and deformability of individual human red blood cells from cord blood of newborn infants and maternal blood," Journal of Biomedical Optics 20(11), 111208 (2015).

69. H. Park, S. H. Hong, K. Kim, S. H. Cho, W. J. Lee, Y. Kim, S. E. Lee, and Y. Park, "Characterizations of individual mouse red blood cells parasitized by Babesia microti using 3-D holographic microscopy," Scientific Reports 5, 10827 (2015).

70. H. Park, M. Ji, S. Lee, K. Kim, Y.-H. Sohn, S. Jang, and Y. Park, "Alterations in cell surface area and deformability of individual human red blood cells in stored blood," arXiv preprint (2015).

71. S. Y. Lee, H. J. Park, C. Best-Popescu, S. Jang, and Y. K. Park, "The Effects of Ethanol on the Morphological and Biochemical Properties of Individual Human Red Blood Cells," PloS One 10(12), e0145327 (2015).

72. J. Yoon, K. Kim, H. Park, C. Choi, S. Jang, and Y. Park, "Label-free characterization of white blood cells by measuring 3D refractive index maps," Biomedical Optics Express 6(10), 3865-3875 (2015).

73. K. Kim, J. Yoon, and Y. Park, "Simultaneous 3D visualization and position tracking of optically trapped particles using optical diffraction tomography," Optica 2(4), 343-346 (2015).

74. Y. Sung, N. Lue, B. Hamza, J. Martel, D. Irimia, R. R. Dasari, W. Choi, Z. Yaqoob, and P. So, "Threedimensional holographic refractive-index measurement of continuously flowing cells in a microfluidic channel," Physical Review Applied 1(1), 014002 (2014).

75. Y. Sung, W. Choi, N. Lue, R. R. Dasari, and Z. Yaqoob, "Stain-Free Quantification of Chromosomes in Live Cells Using Regularized Tomographic Phase Microscopy," PloS One 7(11), e49502 (2012).

76. S. E. Lee, K. Kim, J. Yoon, J. H. Heo, H. Park, C. Choi, and Y. Park, "Label-free quantitative imaging of lipid droplets using quantitative phase imaging techniques," in Asia Communications and Photonics Conference 2014, Optical Society of America, ATh1I.3 (2014).

77. J. Jung, K. Kim, J. Yoon, and Y. Park, "Hyperspectral optical diffraction tomography," Optics Express 24(3), 2006-2012 (2016).

78. W. Krauze, P. Makowski, M. Kujawińska, and A. Kuś, "Generalized total variation iterative constraint strategy in limited angle optical diffraction tomography," Optics Express 24(5), 4924-4936 (2016).

79. R. Zonneveld, G. Molema, and F. B. Plötz, "Analyzing Neutrophil Morphology, Mechanics, and Motility in Sepsis: Options and Challenges for Novel Bedside Technologies," Critical Care Medicine 44(1), 218-228 (2016).

80. J. W. Su, W. C. Hsu, C. Y. Chou, C. H. Chang, and K. B. Sung, "Digital holographic microtomography for high-resolution refractive index mapping of live cells," Journal of Biophotonics 6(5), 416-424 (2013).

81. W.-C. Hsu, J.-W. Su, T.-Y. Tseng, and K.-B. Sung, "Tomographic diffractive microscopy of living cells based on a common-path configuration," Optics Letters 39(7), 2210-2213 (2014).

82. C. Fang-Yen, W. Choi, Y. J. Sung, C. J. Holbrow, R. R. Dasari, and M. S. Feld, "Video-rate tomographic phase microscopy," Journal of Biomedical Optics 16(1), 011005 (2011).

83. J. Yoon, S.-A. Yang, K. Kim, and Y. Park, "Quantitative characterization of neurotoxicity effects on individual neuron cells using optical diffraction tomography," in Asia Communications and Photonics Conference 2015, Optical Society of America, ASu4G.4 (2015).

84. S. Cho, S. Kim, Y. Kim, and Y. K. Park, "Optical imaging techniques for the study of malaria," Trends in Biotechnology 30(2), 71-79 (2011).

85. J. R. Cursino-Santos, G. Halverson, M. Rodriguez, M. Narla, and C. A. Lobo, "Identification of binding domains on red blood cell glycophorins for Babesia divergens," Transfusion 54(4), 982-989 (2014).

86. Y. I. Jeong, S. H. Hong, S. H. Cho, W. J. Lee, and S. E. Lee, "Induction of IL-10-producing CD1d ${ }^{\text {high }} \mathrm{CD}^{+}$ regulatory B cells following Babesia microti-infection," PloS One 7(10), e46553 (2012).

87. M. J. Homer, I. Aguilar-Delfin, S. R. Telford, P. J. Krause, and D. H. Persing, "Babesiosis," Clinical Microbiology Reviews 13(3), 451-469 (2000).

88. A. M. Kjemtrup, and P. A. Conrad, "Human babesiosis: an emerging tick-borne disease," International Journal for Parasitology 30(12-13), 1323-1337 (2000).

89. B. Simon, M. Debailleul, A. Beghin, Y. Tourneur, and O. Haeberle, "High-resolution tomographic diffractive microscopy of biological samples," Journal of Biophotonics 3(7), 462-467 (2010).

90. W.-C. Hsu, J.-W. Su, C.-C. Chang, and K.-B. Sung, "Investigating the backscattering characteristics of individual normal and cancerous cells based on experimentally determined three-dimensional refractive index distributions," Proc. of SPIE 8553, 855310 (2012).

91. C. Zuo, J. S. Sun, J. L. Zhang, Y. Hu, and Q. Chen, "Lensless phase microscopy and diffraction tomography with multi-angle and multi-wavelength illuminations using a LED matrix," Optics Express 23(11), 1431414328 (2015).

92. K. Lee, and Y. Park, “Quantitative phase imaging unit,” Optics Letters 39(12), 3630-3633 (2014). 
93. S. Shin, Y. Kim, K. Lee, K. Kim, Y.-J. Kim, H. Park, and Y. Park, "Common-path diffraction optical tomography with a low-coherence illumination for reducing speckle noise," Proc. of SPIE 9336, 933629 (2015).

94. P. Hosseini, Y. J. Sung, Y. Choi, N. Lue, Z. Yaqoob, and P. So, "Scanning color optical tomography (SCOT)," Optics Express 23(15), 19752-19762 (2015).

95. J. Jung, K. Kim, H. Yu, K. Lee, S. Lee, S. Nahm, H. Park, and Y. Park, "Biomedical applications of holographic microspectroscopy [Invited]," Applied Optics 53(27), G111-G122 (2014).

96. As of Feb 2016, there are two companies provide ODT systems: Tomocube (www.tomocube.com) and Nanolive (www.nanolive.ch).

97. S. M. Popoff, G. Lerosey, R. Carminati, M. Fink, A. C. Boccara, and S. Gigan, "Measuring the Transmission Matrix in Optics: An Approach to the Study and Control of Light Propagation in Disordered Media," Physical Review Letters 104(10), 100601 (2010).

98. H. Yu, T. R. Hillman, W. Choi, J. O. Lee, M. S. Feld, R. R. Dasari, and Y. Park, "Measuring Large Optical Transmission Matrices of Disordered Media," Physical Review Letters 111(15), 153902 (2013).

99. J. Yoon, K. Lee, J. Park, and Y. Park, "Measuring optical transmission matrices by wavefront shaping," Optics Express 23(8), 10158-10167 (2015).

100. H. Yu, J. Park, K. Lee, J. Yoon, K. Kim, S. Lee, and Y. Park, "Recent advances in wavefront shaping techniques for biomedical applications," Current Applied Physics 15(5), 632-641 (2015).

101. A. P. Mosk, A. Lagendijk, G. Lerosey, and M. Fink, "Controlling waves in space and time for imaging and focusing in complex media," Nature Photonics 6(5), 283-292 (2012).

102. C. Park, J. H. Park, C. Rodriguez, H. Yu, M. Kim, K. Jin, S. Han, J. Shin, S. H. Ko, K. T. Nam, Y. H. Lee, Y. H. Cho, and Y. Park, "Full-Field Subwavelength Imaging Using a Scattering Superlens," Physical Review Letters 113(11), 113901 (2014).

103. T. Čižmár and K. Dholakia, "Exploiting multimode waveguides for pure fibre-based imaging," Nature Communications 3, 1027 (2012).

104. K. Kim, K. S. Kim, H. Park, J. C. Ye, and Y. Park, "Real-time visualization of 3-D dynamic microscopic objects using optical diffraction tomography," Optics Express 21(26), 32269-32278 (2013).

105. J. W. Su, Y. H. Lin, C. P. Chiang, J. M. Lee, C. M. Hsieh, M. S. Hsieh, P. W. Yang, C. P. Wang, P. H. Tseng, Y. C. Lee, and K. B. Sung, "Precancerous esophageal epithelia are associated with significantly increased scattering coefficients," Biomedical Optics Express 6(10), 3795-3805 (2015).

106. S. Lee, K. Kim, A. Mubarok, A. Panduwirawan, K. Lee, S. Lee, H. Park, and Y. Park, "High-Resolution 3-D Refractive Index Tomography and 2-D Synthetic Aperture Imaging of Live Phytoplankton," Journal of the Optical Society of Korea 18(6), 691-697 (2014).

107. S. Lee, K. Kim, Y. Lee, S. Park, H. Shin, J. Yang, K. Ko, H. Park, and Y. Park, "Measurements of morphology and refractive indexes on human downy hairs using three-dimensional quantitative phase imaging," Journal of Biomedical Optics 20(11), 111207 (2015).

108. K. Kim, J. Yoon, and Y. Park, "Large-scale optical diffraction tomography for inspection of optical plastic lenses," Optics Letters 41(5), 934-937 (2016).

\section{Introduction}

Three-dimensional (3-D) optical microscopy techniques have been an invaluable tool in modern biological and medical sciences. They have been utilized to investigate mechanisms and dynamics of biological cells and to provide insights to aid in the diagnosis of diseases [1]. For recent half a century, we have witnessed the developments of various 3-D optical microscopy techniques and their applications in cell biology and medicine; from confocal microscopy [2] to multiphoton microscopy [3], and to super-resolution nanoscopy [4].

Among them, optical diffraction tomography (ODT) has recently created and demonstrated a wealth of possibilities for 3-D imaging of live cells. ODT is an interferometric technique which measures the 3-D refractive index (RI) distribution of optically transparent samples such as biological cells. ODT is analogous to X-ray computed tomography (CT), except that it uses visible light instead of X-ray. Recently, ODT has gained significant interest, and the applications using ODT has rapidly expanded due to the following advantageous:

- ODT does not require the use of exogenous labeling agents or dyes, such as fluorescence proteins, organic/inorganic dyes, and quantum dots. In ODT, RI, an intrinsic optical parameter of material, is utilized as an imaging contrast. ODT eliminates the complicated sample preparation processes and overcomes the limitations of labeling agents or dyes such as photobleaching or phototoxicity.

- In ODT, the measurements of RI provide quantitative imaging capability. The values of RI can be translated into various useful parameters such as protein concentrations and cellular dry mass.

- The instrumentational requirements for ODT are relatively simple and inexpensive. Unlike 3-D nonlinear optical microscopy techniques such as 
multiphoton microscopy or Raman microscopy, ODT utilizes low-power continuous-wave lasers for illumination and conventional image sensors such as CCD or CMOS.

ODT was first theoretically proposed in 1969 by E. Wolf [5] and followed by geometrical interpretation by Dändliker \& Weiss [6]. From the late 70s, ODT has been experimentally demonstrated by early works [7-12]. Recently, there have been significant technical advances in ODT thanks to the advancements of laser sources, detecting devices, and computing powers. Also, the limitations and challenges of using labeling agents in 3-D cell imaging have become significant issues in several applications. For these reasons, during the last few years, the field of ODT has started to expand to the various fields of applications, ranging from biophysics, cell biology, hematology, to infectious diseases.

Here, we summarize the recent advances in the developments of ODT techniques and discuss important applications of ODT in the fields of biology and medicine. This review is organized as follows: Section 2 briefly discusses the principles of ODT including optical instrumentations and reconstruction algorithms; Sections 3 summarizes the applications of ODT for the study of cell pathophysiology; Finally, perspectives for future developments and applications are discussed in Section 4.

\section{Materials and methods}

In this section, the principles of ODT are discussed: various experimental configurations for ODT measurements and reconstruction algorithms are summarized. Further information on theoretical principles and experimental configurations for various ODT techniques can also be found elsewhere [13-16].

\subsection{Experimental setup}

ODT solves an inverse problem of light scattering by a weakly scattering object. Typically, 3-D RI distribution of a weakly scattering sample or so-called a phase object is reconstructed from the measurements of multiple 2-D holograms of the object obtained with various illumination angles. It is analogous to X-ray CT (Fig. 1), in which 3-D absorption distribution of a human body is reconstructed via a filtered back projection algorithm, from the measurements of multiple 2-D X-ray images of the object obtained with various illumination angles. Both the ODT and X-ray CT shares the same governing equation - Helmholtz equation, and thus the principle of reconstruction algorithms is also identical.

Essentially, the optical setup for ODT consists of two parts: the illumination or sample modulation unit and the optical field recording unit. The optical field, composed of both the amplitude and phase information about a sample, is recorded employing the principle of interference. Diverse configurations are available for the optical field recording unit, including off-axis interferometry, phase shifting interferometry, or using transport of intensity equation. In-depth summaries on various 2-D field measurements techniques and field retrieval algorithms can be found in elsewhere [17-20].

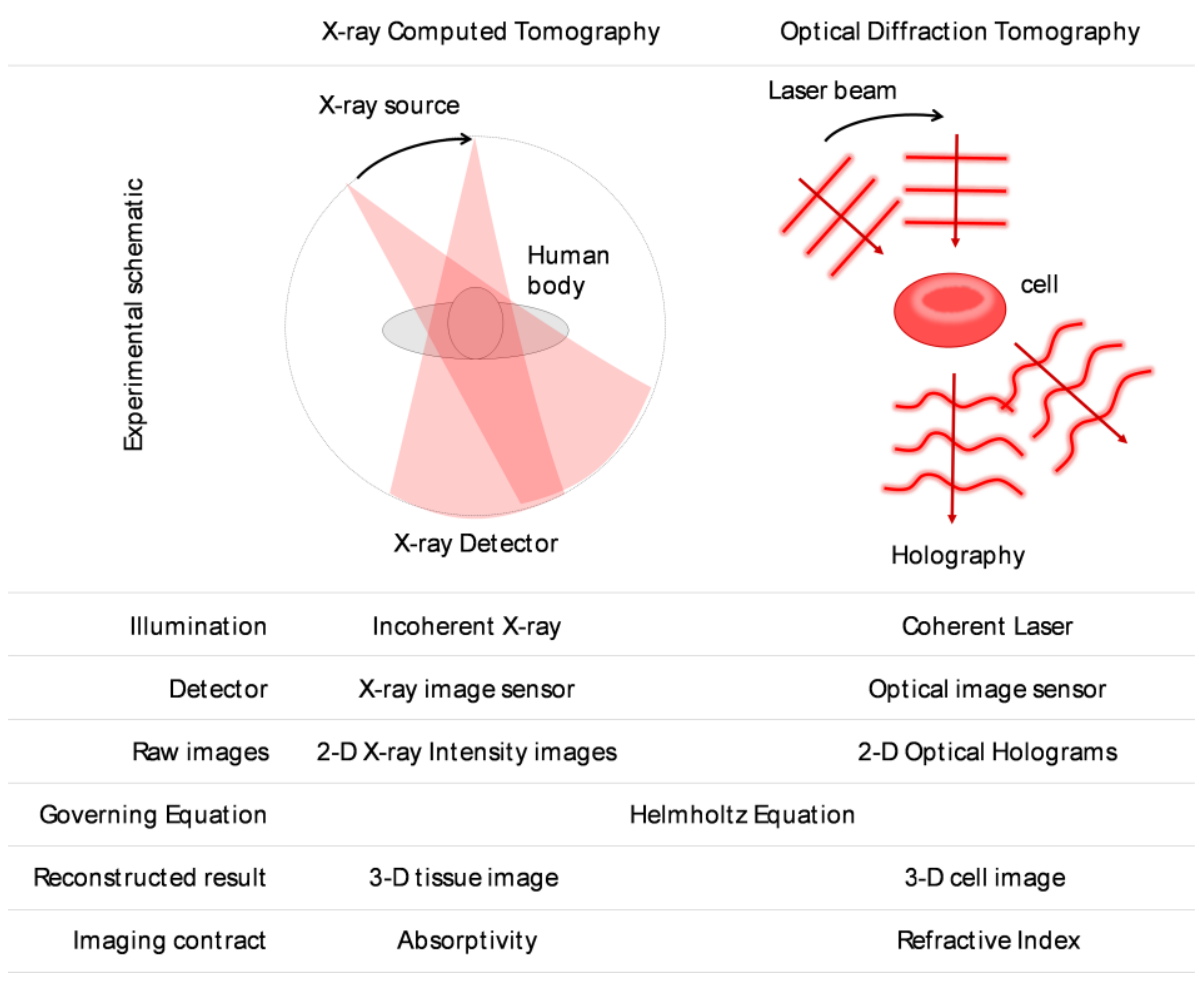

Fig. 1 Schematic diagram of the experimental setup. 
To control illumination beams impinging onto a sample, various techniques have been proposed, which can be classified into illumination scanning [21-24] and sample rotating schemes [25-27].

\subsubsection{Illumination scanning ODT}

For systematic controlling the angle of illumination beams, various types of beam rotators have been employed as depicted in the Figs. 2 (a1-a3). Typically, galvanometer-based rotating mirrors have been used as a beam rotator, which controls the angle of the illumination beam impinging onto a sample by tilting mirrors of the galvanometer located at the conjugate plane of the sample [Fig. 2(a1)]. Due to the use of mirrors, beam scanning with a galvanometer can be executed with minimal laser power loss. The use of the galvanometer, however, is inherent in mechanical instability including position jittering induced by electric noise and positioning error at high voltage values caused by the nonlinear response. Moreover, due to the geometry of the dual-axis galvanometer, rotational surfaces for two independent axes cannot be exactly conjugated to the sample plane, which causes unwanted additional quadratic phase distribution on the illumination beam. To overcome this optical misalignment, placing two single-axis galvanometers at the separate conjugate plane relayed by additional 4-f lenses can be used, but it inevitably requires a bulky optical system that may cause additional phase instability due to long optical paths.

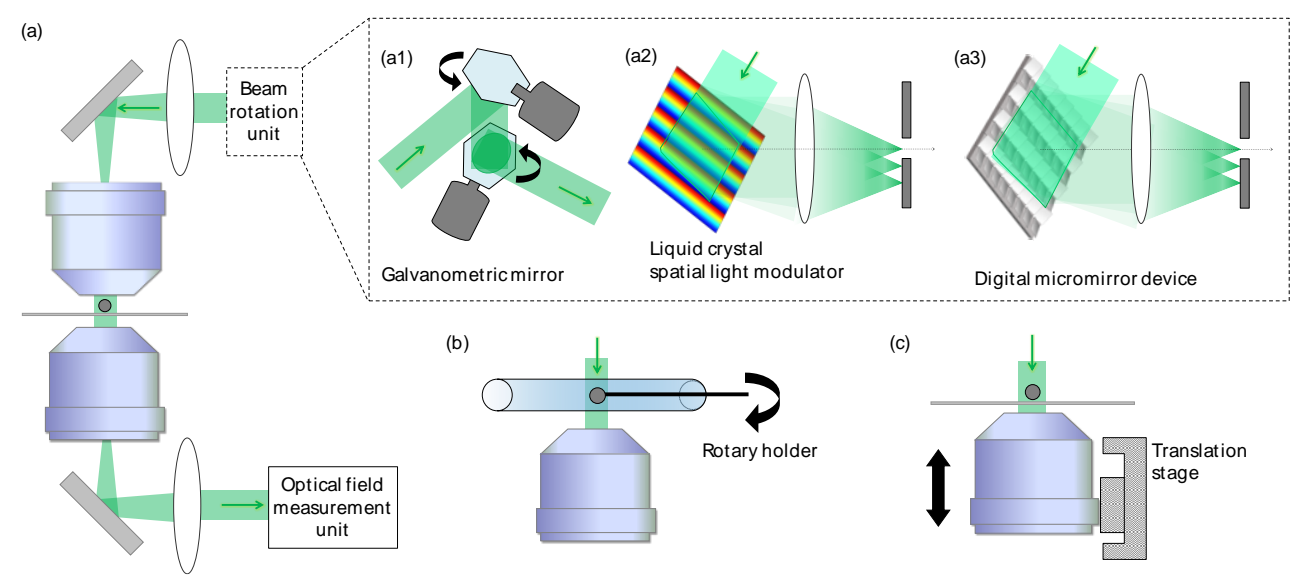

Fig. 2 Experimental setups for standard ODT techniques. (a) Beam scanning methods using a beam rotator such as (a1) a dual-axis galvanometer mirror, (a2) a liquid crystal spatial light modulator, and (a3) a digital micromirror device. (b) A sample rotating method using a rotary holder, and (c) axial scanning of objective lens using a translaional stage.

Recently, a spatial light modulator (SLM) using liquid crystals was utilized as a beam rotator [28]. To control the angle of the illumination beam, the SLM located at the conjugate plane to the sample displays wrapped linear phase ramp. Then, a plane wave with a desired propagating direction can be generated from the first-order diffracted beam while unwanted diffracted beams are blocked by spatial filtering [Fig. 2(a2)]. Since the use of the SLM does not contain any mechanically moving part, the ODT measurements can be performed with high stability. Also, the SLM can correct the wavefront distortion of an illumination beam to generate clearly plane waves at the sample plane, which enhances the accuracy of ODT measurements. However, due to the limited diffraction efficiency of the SLM and the spatial filtering, available illumination beam power significantly decreases. Furthermore, the slow innate response of liquid crystal realignment in the SLM slows down the scanning rate of the illumination beam, and expensive costs of an SLM may also limit wide applications in biological fields.

More recently, a digital micromirror device (DMD) was employed as a beam rotator for the ODT technique with high speed and stability [29, 30]. The DMD consists of hundreds of thousands of switchable micromirrors, which can be independently controlled between on and off states with ultra-high modulation speed reaching few tens of $\mathrm{kHz}$. To steer the angle of the illumination beam, the DMD located at the conjugate plane to the sample displays a binary pattern, called the Lee hologram [31] to generate a plane wave with a desired illumination angle [Fig.2 (a3)]. Similar to the use of an SLM, the illumination control with a DMD can be performed in high stability, and also able to correct wavefront distortion. Furthermore, the use of a DMD is cost efficient and enables fast illumination control due to the ultra-fast refreshing speed of a DMD. Because binary intensity holograms are projected, there is a limitation in the dynamic range of phase modulation and a beam size of the illumination with a DMD. Also, undesired diffraction from a DMD causes speckle noise and reduction in beam power. 


\subsubsection{Sample rotation $O D T$}

Instead of illumination control, a sample of interest can be rotated, and the diffracted light fields at various rotation angles can be used for the reconstruction of 3-D RI map of the sample [Fig. 2(b)]. Contrast with the beam scanning method, a whole range of viewing angles can be addressed in the sample rotating method. To experimentally achieve the rotation of a sample, a rotary microcapillary is typically utilized [25-27]. Compared to illumination scanning ODT, which requires complicated optical setups for beam rotators, sample rotation ODT employs relatively simple optical setups, and reconstructed tomograms exhibit isotropic spatial resolution along the lateral and axial directions. Mechanical rotation of samples, however, limits data acquisition speed. Also, perturbation occurred during mechanical rotation, called radial run-out [32], and field distortion due to the refraction from the cylindrical microcapillary require additional numerical correcting algorithms [33]. More importantly, the sample rotation method can cause deformation of live biological cells because of the viscoelasticity of cells, possibly resulting in artifacts in reconstructed tomograms.

Recently, to avoid the perturbations and the refraction due to the use of the microcapillary, sample rotation methods exploiting optical trapping have been proposed [34-36]. Optical tweezers ware used to rotate RBCs in a microfluidic channel in order to measure 3-D morphology of cells [34]. Recently, optical force rotated a yeast cell [35] and a myeloid precursor cell [36] with precise rotation angles, from which 3-D RI distributions of rotating samples are reconstructed. Sample rotation with optical trapping, however, may induce morphological deformation of biological cells during rotation, and applications are limited to samples which can be floated; it would be challenging to measure optical adherent cells or soft cells using the optical trapping method.

\subsubsection{Other methods}

Alternatively, axial scanning of either an objective lens or a sample stage has also been utilized for reconstructing 3-D RI distribution of biological specimens [Fig. 2(c)] [37-39]. These methods can simplify optical setups, but exhibits limited axial resolutions. The use of low-coherence sources or the deconvolution has been reported for better axial resolutions [37-39]. Also, acquisition speed is also limited because mechanical scanning of an objective lens or a sample stage is slower than the use of galvanometric mirrors or DMDs. Also, scanning wavelengths of the illumination beam has also been utilized for measuring 3-D RI maps of samples [40, 41]. Recently, lens-free holographic and phytography techniques have been introduced which simplifies optical setup for ODT $[42,43]$.

\subsection{Reconstruction procedures for ODT}

In order to reconstruct the 3-D RI distribution of biological cells from measured multiple 2-D optical fields, several computational steps are required: (i) the application of appropriate reconstruction algorithms for solving an inverse scattering problem; (ii) the application of weak-scattering approximations depending on the scattering property of a sample, and (iii) regularization processes to fill information caused from limited measurements.

\subsubsection{The reconstruction algorithms: projection and diffraction tomography algorithm}

The reconstruction algorithm can be classified into two groups: the diffraction algorithm and the projection algorithm. They are both inverse scattering solvers, but the difference is whether the effects of light diffraction is considered or not. Generally, ODT implies the reconstruction of 3-D RI maps using the diffraction algorithm. A few early works employed the projection algorithm for the study of cells [22]. However, it has been shown that individual biological cells exhibit significant light diffraction, especially for cells with complex internal structures [38].

Figure 3 presents the working scheme of the tomogram reconstruction algorithm employing either the projection algorithm and the diffraction algorithm. Complex optical fields of a polystyrene (PS) bead with the diameter of $10 \mu \mathrm{m}$ were measured with various illumination angles [Fig. 3(a)], and the 2-D Fourier spectra of the measured optical fields were mapped into the 3-D Fourier space according to either the projection algorithm [Fig. 3(b)] or the diffraction algorithm [Fig. $3(\mathrm{c})$ ]. In order to systematically compare the quality of the tomogram using either reconstruction algorithms, we performed experiments with two data sets; single PS bead placed at the focal plane and two PS bead placed at the different planes.

The projection tomography assumes that each 2-D optical field image corresponds to the projection of the 3-D object at certain illumination angle and light diffraction inside samples is ignored. In other words, measured optical phase delay is assumed to be an integration of RI values along a straight light. In this case, the 2-D Fourier spectrum of the measured optical field at a certain illumination angle is identical to the inclined planar slice of 3-D Fourier spectra of the object, according to the Fourier slice theorem [13], as shown in Fig. 3(b). Thus, 3-D Fourier spectra can be mapped by a series of 2-D Fourier spectra which are obtained from the measured multiple 2-D optical fields with various incidence angles. Finally, the inverse Fourier transform of the mapped 3-D Fourier spectra provides the reconstruction of the 3-D RI distribution of the sample [Fig. 3(d)].

Implementation of the projection tomography algorithm is straightforward: applying inverse Radon transformation to the series of the measured optical 
fields with various incident angles, which is called as a sinogram, directly provides the reconstruction of a 3-D RI map. The projection algorithm is valid when the wavelength of illumination is much smaller than the size of a sample. Thus, the wave propagation can be treated as projection. In X-ray CT, this condition is valid for general conditions, but this limits applications in the optical wavelengths. In the visible wavelengths, the sizes of the cellular components are compatible with the wavelengths of an illumination beam. Thus, light diffraction effects inside cells are not negligible so that the application of the projection algorithm deteriorates the image quality of the reconstructed tomograms, depending on samples. Although the projection algorithm has been successfully applied to the tomographic RI measurements of optical elements [25, 44] and biological samples [22, 45-47], it was shown that effects of light diffraction cannot be ignored even at the individual cell levels [38]. Thus, the use of the diffraction algorithm is recommended except for some special cases when the size of a sample is much large than the wavelength and RI contrast in a sample is negligible. Otherwise, the use of the projection algorithm may result in incorrect RI values and distorted shapes in the reconstructed tomograms. These unwanted artifacts become severe for objects located at defocused planes or for samples with a high optical phase delay.
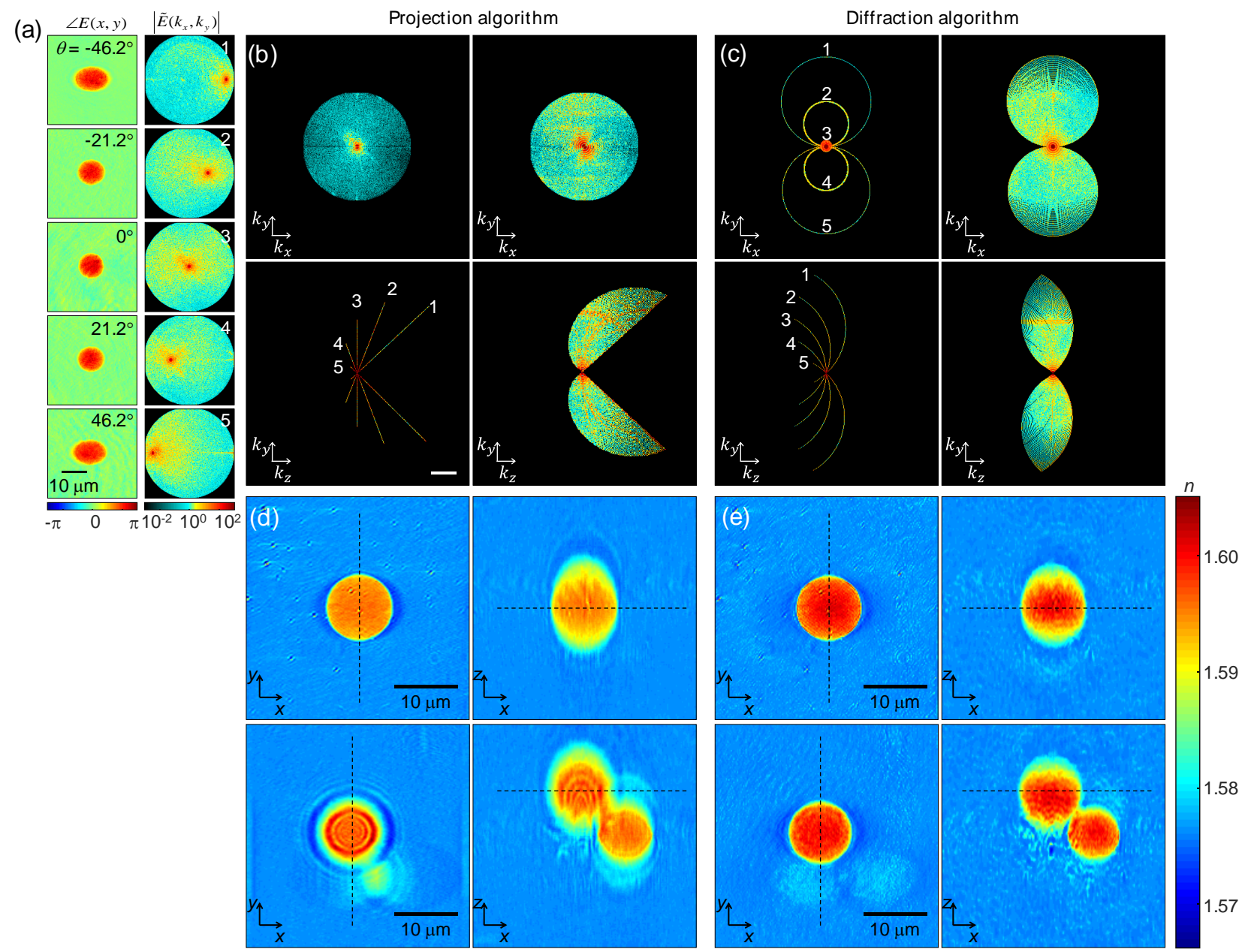

Fig. 3 The principle of optical projection and diffraction tomography algorithm. (a) Quantitative phase images of a polystyrene bead with the diameter of $10 \mu \mathrm{m}$ illuminated at various incidence angles, and corresponding 2-D Fourier spectra of complex optical fields. (b-c) Object functions in the 3-D Fourier space mapped by (b) projection tomography and (c) diffraction tomography. (d-e) Cross-sectional slices of the 3-D RI distribution of the polystyrene bead reconstructed by (d) projection tomography and (e) diffraction tomography.

The diffraction algorithm considers light diffraction inside a sample by solving the Helmholtz equation for the incident and scattered optical fields. Thus, the 3-D RI distribution of a sample can be obtained with more precise shapes and RI values of a sample than the case with the projection algorithm [5]. According to Fourier diffraction theorem, 2-D Fourier spectra of an optical field at a certain illumination angle is mapped onto the surface of a hemisphere called Ewald sphere. The center position of the Ewald sphere is translated from the origin in the 3-D Fourier space by the distance and direction corresponding to the $\mathrm{k}$ vector, or spatial frequency, of the incident angle [Fig. 3c]. Mapping Fourier spectra of multiple optical fields at various 
incident angles fill the 3-D Fourier space and 3-D inverse Fourier transform of the 3-D Fourier space results into the reconstruction of the 3-D RI distribution of a sample [Fig. 3e]. The detailed and rigorous mathematical derivations of the diffraction algorithm can be found elsewhere [5, 13, 21, 48].

Figures 3(d) and 3(e) clearly show that tomograms reconstructed by the diffraction algorithm provide more precise RI values and shapes of samples than the case with the projection algorithm, especially when optical fields of a sample are severely distorted either by highly scattering internal structures or when a target object is located at the defocused plane. The upper panel of Figs. 3(d)-(e) shows the tomogram of a PS bead placed at the focal plane. Both tomogram reconstruction methods exhibit uniform RI distribution at the focal plane. However, the RI distribution along the optic axis reconstructed by the projection method shows axially elongated shapes due light diffraction from the top of the bead. The effects of light diffraction on tomogram reconstruction become more severe when the PS bead is placed at the defocused plane [the lower panel of Figs. 3(d)-(e)]. For the projection method, the PS bead located at the defocused plane exhibits unwanted fringe patterns due to light diffraction, while the diffraction method reconstructs uniform RI distribution of PS beads independently of the axial positions. Quantitative comparisons of tomogram reconstruction quality between the projection and the diffraction algorithm have been reported with various types of samples, including multimode fibers [49], colloidal particles [48], and malaria-infected human red blood cells (RBCs) [38].

\subsubsection{Weak scattering approximation: Born vs. Rytov approximations}

Finding the 3-D scattering potential, or the 3-D RI map, of a sample from the measured multiple 2-D optical fields, is an ill-defined inverse problem, which cannot be directly solved. However, under the first-order weak scattering approximation, the Helmholtz equation can be linearized, and the analytic solution for this inverse problem can be found. The weak scattering approximation for the linearization includes the Born or Rytov approximation [5, 13, 50].

The first-order Born approximation assumes that the scattered optical field from a sample is significantly weaker than the incident optical field, and assumes the total optical fields inside the sample as the incident optical field.

Specifically, the first-order Born approximation is valid when the total optical phase delay of a sample $\Delta \varphi$ is smaller than $\pi / 2$ [48]. However, the optical phase delay of most biological cells exceeds $\pi$ rad. For example, the cell thickness of $10 \mu \mathrm{m}$ and the RI contrast between a cell and a medium greater than 0.03 are already beyond the valid regime of the first-order Born approximation. As shown in Figs. 4a and 4c, the Born approximation successfully reconstructs 3-D RI distribution of a $5-\mu \mathrm{m}$ PS bead $(\Delta \varphi=0.41 \pi \mathrm{rad}$.) whereas it fails to reconstruct for a $10-\mu \mathrm{m}$ PS bead $(\Delta \varphi=0.83 \pi \mathrm{rad}$.$) . In contrast, the first-order Rytov$ approximation considers the total optical field as a complex amplitude, and assumes that the spatial variations of the complex amplitude in the wavelength scales are smaller than 1 [51]. For that reason, the validity of the first-order Rytov approximation is independent of the total optical phase delay as:

$$
\Delta n \gg\left(\frac{\lambda \nabla \varphi_{\mathrm{s}}}{2 \pi}\right)^{2},
$$

where $\Delta n$ is the RI contrast between a cell and a medium and $\nabla \varphi_{s} \mathrm{~s}$ is the spatial variations of the complex amplitude. The above relation can be deduced to $\Delta n \ll n_{m}$ for the weak scattering sample where $\nabla \varphi_{s}$ is linearly proportional to $\Delta n$ [52]. As shown in Figs. $4 \mathrm{~b}$ and $4 \mathrm{~d}$, the first-order Rytov approximation remains valid whenever the spatial gradient of the optical phase is not significant. Because most biological cells have a small spatial gradient of RI distribution, especially when cells are submerged in a medium, the first-order Rytov approximation is suitable for the tomographic reconstruction.

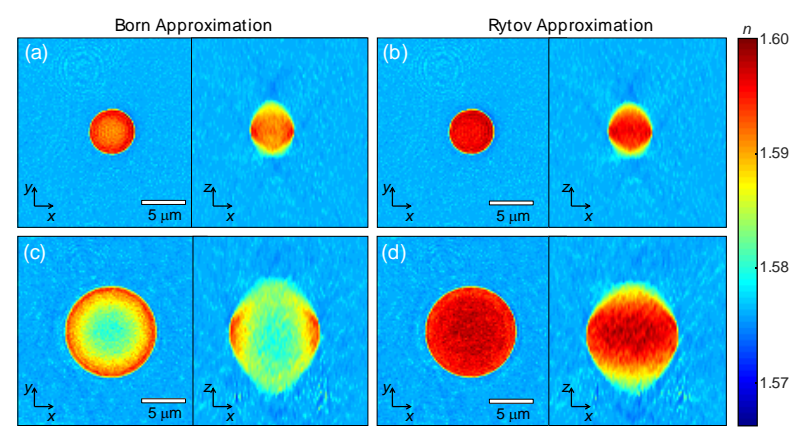

Fig. 4 Comparison between (a \& c) the first-order Born and $(b \& d)$ the first-order Rytov approximation in ODT. (a-b) Cross-sectional slice of the 3-D RI distribution of a 5 - $\mu$ m-diameter polystyrene bead with (a) Born and (b) Rytov approximation. (c-d) Same as (ab) for a $10-\mu \mathrm{m}$-diameter polystyrene bead.

\subsubsection{Regularization process}

Unlike X-ray CT where full $360^{\circ}$ angle of illumination is possible, the ODT techniques employing the beam scanning method has a limited angle range of illumination, which is mainly determined by the numerical aperture (NA) of objective lenses. Consequently, in the Fourier space, there exists the inaccessible region corresponding to the spatial frequencies beyond the maximum spatial frequencies of the imaging system. This issue due to the inaccessible region is called as the missing cone problem because the shape of the inaccessible region resembles a cone. This missing cone problem causes shape elongation and under-estimated RI values in reconstructed tomograms $[53,54]$. Although the missing cone problem is unavoidable for the ODT techniques with the beam 
scanning, it can also occur in the cases with the sample rotation method; incomplete rotation of a sample results in a similar situation comparable to the limited numerical aperture in the beam scanning method. Rotating a sample around one axis also generates a missing part in the Fourier space which shape resembles an apple core [55].

To relieve the missing cone problem and enhance the quality of tomogram reconstruction, several iterative algorithms have been proposed to fill the unassessed information based on a priori information about an object such as the non-negativity constraint algorithm, the edge-preserving regularization, and the totalvariation regularization. Figure 5 shows comparisons of the 3-D RI distribution of a hepatocyte reconstructed without the regularization process [Fig. 5(a)], which is reproduced from Ref. [56]. For comparison, the 3-D RI maps of the hepatocyte obtained using the various regularization methods including the non-negativity constraint, the edge-preserving regularization, and the total-variation regularization algorithm are presented in Fig. 5(b)-(d), respectively. Detailed information about these iterative algorithms and comparative studies can be found elsewhere $[56,57]$.

Recent works have shown that this missing cone problem can be overcome alternatively. Complex deconvolution using a predefined coherent transfer function (CTF) corrects aberration induced by the missing cone problem and achieves sub-100-nm lateral resolution using objective lenses with high numerical aperture [23]. The missing cone problem can also be relieved using a machine-learning algorithm based on the beam propagation method [58].

\subsubsection{Spatial Resolution in $O D T$}

Theoretically, the spatial resolution of ODT is defined as the maximum spatial frequency in reconstructed Fourier spectra. This maximum spatial frequency is mainly determined by the wavelength of light $\lambda$, the numerical aperture of an imaging system $N A$, the RI value of immersion media $n_{i}$, and the optical configuration for ODT [21], which is summarized in Table 1.
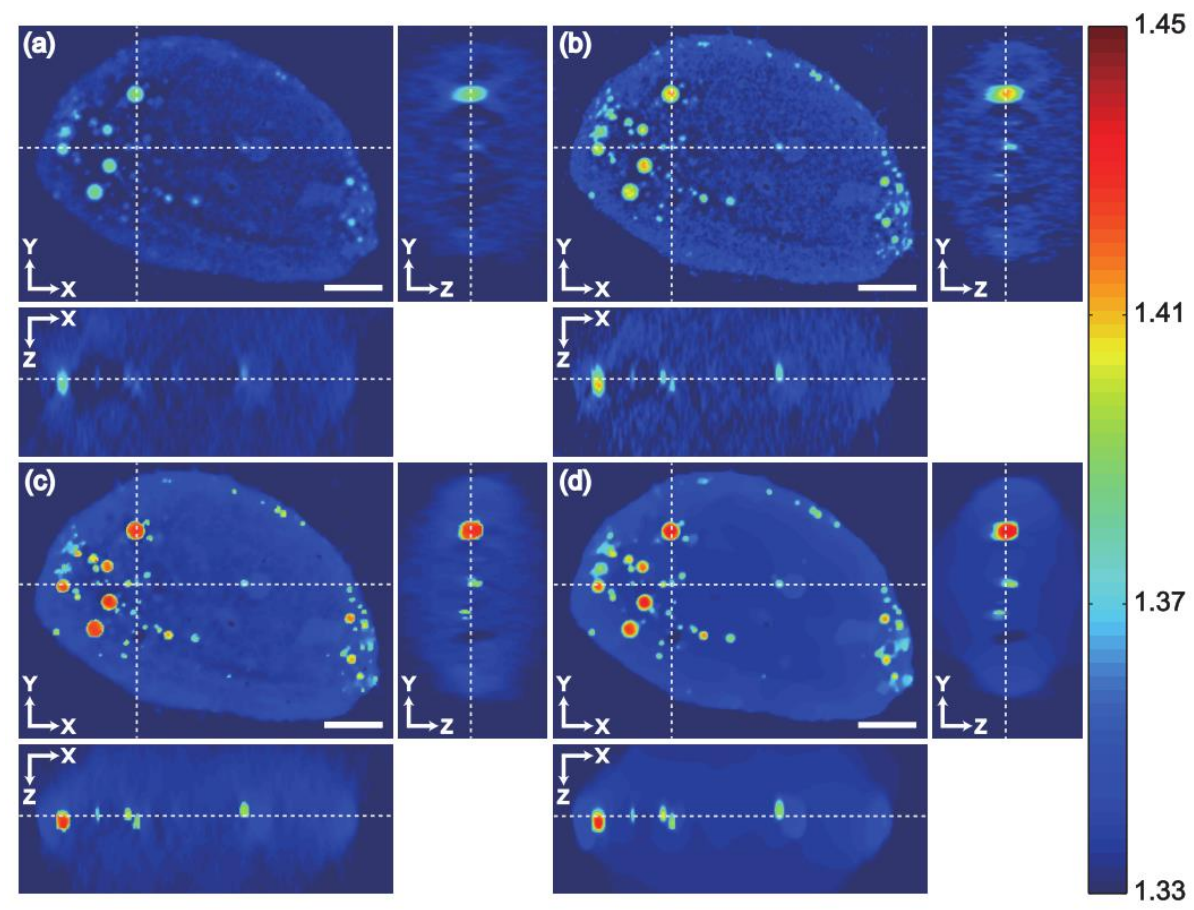

Fig. 5 Comparison of 3-D RI distribution of a hepatocyte reconstructed by (a) direct Fourier transform, (b) nonnegativity constraint, (c) edge-preserving regularization, and (d) total-variation regularization algorithm. Reprinted from Ref. [56] with permission.

Table 1 Lateral and axial resolution in ODT.

\begin{tabular}{ccc}
\hline & Lateral resolution & Axial resolution \\
\hline The sample rotation method & $\frac{\lambda}{2 N A}$ & $\frac{\lambda}{2 N A}$ \\
\hline The beam scanning method & $\frac{\lambda}{4 N A}$ & $\frac{\lambda}{2} \frac{1}{n_{i}-\sqrt{n_{i}^{2}-N A^{2}}}$ \\
\hline
\end{tabular}


The axial resolution of illumination-scanning ODT is very sensitive to NA and ni. For instance, with a 532nm-wavelength laser, the lateral resolution using an objective lens with $\mathrm{NA}=0.9$ and NA $=0.45$ is $148 \mathrm{~nm}$ and $296 \mathrm{~nm}$, respectively, but the axial resolution using the same objective lens corresponds to $472 \mathrm{~nm}$ and 2.49 $\mu \mathrm{m}$, respectively. In a recent work, it was theoretically proposed that ODT can provide isotropic lateral and axial resolution of $\lambda /(4 N A)$ by combining sample rotation and illumination scanning [59]. Since the size of most biological cells ranges from few micrometers to tens of micrometer, the spatial resolution of ODT is sufficient for resolving structures and biochemistry inside biological samples, but ODT has difficulties for resolving small subcellular organelles including ribosomes, lysosomes, and small vesicles which sizes are sub-100 nm.

\section{Study of cell pathophysiology using ODT}

Due to the aforementioned advantages, there is an increasing number of studies employing ODT for the study of cell pathophysiology. Label-free visualization of live cells has a potential for the investigation of functions and mechanisms at the individual cell level. The following section summarizes physiological parameters of cells accessible with ODT and then highlights the representative pathophysiological study of cells with ODT techniques.

\subsection{Quantitative physiological parameters of cells accessible with ODT}

Quantitative 3-D imaging capability of ODT enables the retrieval of various physiological parameters, including morphological and biochemical parameters.

3-D RI maps of a cell directly provide several morphological parameters. First, from distinct boundaries in a 3-D RI map provide information about the volume $V$ and surface area $A$ of cells and subcellular organelles. From the retrieved values of $V$ and $A$, sphericity $\psi$, a dimensionless parameter indicating how much an object is spherical, can be calculated as $\psi=\pi^{\frac{1}{3}}\left(6 V^{\frac{2}{3}}\right) / A$. To address the cellular or subcellular boundaries from measured 3-D RI maps, isosurfaces can be extracted [38]. Recently, it was shown that these boundaries can be effectively retrieved using the multidimensional transfer function, in which both the spatial gradient of RI and the RI values are simultaneously utilized [30]. These morphological features can be potentially valuable for investigating structural characteristics of cells and effects of internal and external stimuli on cellular functions such as osmotic stress and drug treatment.

The values of RIs can provide biochemical information about cells. For example, the values of RI can be translated into the local concentration in the cell cytoplasm. Because the RI values of a solution are linearly proportional to the concentration of solutes, the RI values of cell cytoplasm can be quantitatively converted into the local cytoplasmic concentration. Measured RI value of a biological sample $n$ is linearly proportional to dry mass density $\mathrm{C}$ as $n=n_{m}+\alpha C$, where $n_{m}$ is the RI value of a medium surrounding a cell, $\alpha$ is RI increment [60, 61]. Furthermore, together with the retrieved value of a cell volume, the dry mass the total mass of non-aqueous materials inside a cell can also be calculated from the concentration, because mass is a product of concentration and volume. The values of $\alpha$ are similar regardless of protein types; most amino acids exhibit very similar values of $\alpha$ [62-65]. Because biological cells mostly consist of proteins (amounts of carbohydrates, lipids, and nucleic acids can be ignored), the RI increment for cells can be assumed as a constant when calculating the dry mass and the dry mass density of biological cells. Recently, there have been several studies using ODT to investigate the physiology of biological cells, including red blood cell (RBC) [29, 30, 38, 66-71], white blood cell (WBC) [27, $30,72,73]$, and various eukaryotes $[27,30,48,67$, 74-78]. The following sections highlight representative work.

\subsection{Studies with human red blood cells}

Human RBCs or erythrocytes have a donut-like shape with a diameter of $8.5 \mu \mathrm{m}$ and a thickness of $2.5 \mu \mathrm{m}$, whose function is to carry oxygen from lungs to tissues. RBCs exhibit remarkable deformability, which enables them to pass through small capillaries even narrower than RBC sizes. Physiologically relevant cell indices for RBCs include cell volume, surface area, sphericity, Hemoglobin $(\mathrm{Hb})$ content, and $\mathrm{Hb}$ concentration. In the clinic, some of these RBC indices are routinely measured with a complete blood count (CBC) machine which exploits various analytical methods such as light scattering, electrical impedance, and fluorescence.

Employing ODT, these RBC indices can be quantitatively and precisely measured at the individual cell level, whereas conventional CBC machines measure averaged values from many cells [66]. Morphological parameters such as cell volume, surface area, and sphericity are retrieved from 3-D RI maps of RBCs. Cytoplasmic $\mathrm{Hb}$ concentration is directly converted from measured RI values, and $\mathrm{Hb}$ contents can be calculated from $\mathrm{Hb}$ concentration and cell volume because RBC cytoplasm mainly consists of $\mathrm{Hb}$ solution without a nucleus or other subcellular organelles. Several pathophysiological states in RBCs associated with morphology have been investigated using ODT, including malaria disease [Fig. 6(a)] and babesiosis [Fig. 6(b)], iron-deficiency anemia [Fig. 6(c)] and hereditary spherocytosis (Fig. 6(d) and [66]). Recently, ODT was employed for the characterization of RBCs from cord blood of newborn infants [68]. This study reported that cord RBCs exhibit smaller cell surface area and volume, but the elevated level of $\mathrm{Hb}$ contents, compared to adult RBCs.

Recently, Lee et al. investigated the in-vitro effects of ethanol on morphological and biochemical properties 
of human RBCs using ODT [71]. In this work, no significant alterations have been observed in shapes and $\mathrm{Hb}$ contents of RBCs, whereas significant enhancements in dynamic membrane fluctuation have been measured which indicates enhanced deformability and membrane fluidity [71]. More recently, properties

\section{(a) Plasmodium falciparum}

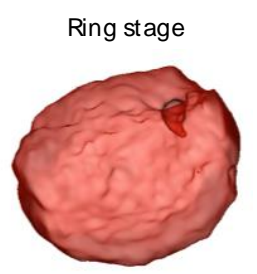

(b) Babesia microti

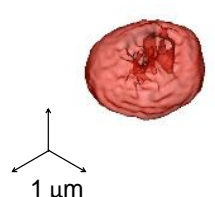

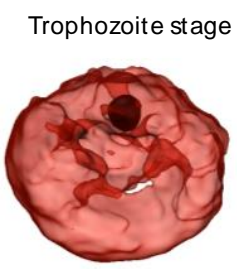

(c) iron deficiency anemia

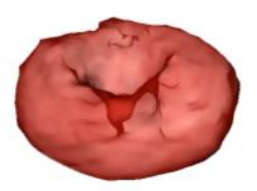

of individual RBCs in stored blood and the effects of a blood preservation solution, CPDA-1, have been systematically investigated by measuring 3-D RI maps of individual RBCs with various blood storage durations and conditions [70].

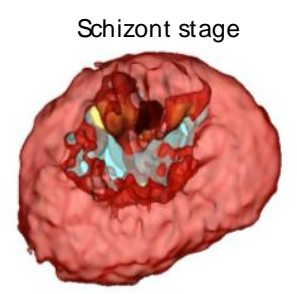

(d) hereditary spherocytosis

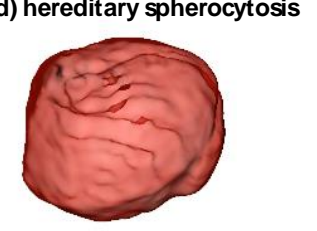

Fig. 6 3-D isosurfaces of representative RBCs under various pathological conditions: (a) RBCs parasitized by Plasmodium falciparum at the ring (left), trophozoite (center) and schizont (right) stage, respectively. (b) Mouse RBC parasitized by Babesia microti. (c) RBC from a patient with iron deficiency anemia. (d) RBC with a patient with hereditary spherocytosis. Arrows indicate $1 \mu \mathrm{m}$ along the $x, y$, and $z$-axis.

\subsection{The study of white blood cells}

WBCs are classified into several cell types including B cells, $\mathrm{T}$ cells, and macrophages; their roles are defending the host against harmful pathogens, abnormal cells, and other invaders. It is well known that WBCs show alterations in morphological and biochemical characteristics in response to immune stimuli, such as bacterial infection and sepsis [79].

ODT has been applied for label-free quantitative analysis of several WBCs and demonstrated potential for the study of WBCs. Yoon et al. have shown the characterization of morphological and biochemical properties of individual lymphocytes and macrophages [Fig. 7(b)]. In particular, alterations in these parameters of WBCs in response to immune stimulus and internalization of a microsphere via phagocytosis have also been investigated using ODT [27, 72]. Recently, ODT in combination with the optical tweezers technique has shown potential for the investigation of the cellular response of macrophages to external mechanical stimuli [73]. When a microscopic particle, trapped and optically manipulated with the optical tweezers, was approached to a macrophage, the deformation of the cell upon this mechanical stimulus has been precisely visualized from the measured 3-D RI maps over time.

\subsection{Physiology of other eukaryotic cells}

The applications of ODT for the study of eukaryotic cells are in the beginning stage, and only a few recent works have been reported. Nonetheless, ODT has shown great potential for the visualization of eukaryotic cells, because it provides not only physical and biochemical properties of eukaryotes, it can also effectively visualize intracellular organelles from the measured 3-D RI map. It has been shown that ODT is capable of visualizing plasma membrane, nucleus, nucleoli, chromosomes, and internal vesicles [27, 74, $80,81]$. Using dual wavelength ODT using visible and ultraviolet wavelengths, Sung et al. have shown that the dry mass of condensed chromosomes, as well as entire cells, can be measured and quantified with ODT, by measuring the 3-D RI maps of HT-29 and T84 cells in metaphase [75]. In particular, this work also presented that the use of the diffraction algorithm with the regularization process provides more precise measurements in compared to one obtained with the projection algorithm, which is confirmed by the 3-D image obtained by laser scanning confocal fluorescence microscopy. Kuś et al. have measured the 3-D RI maps and analyzed the morphological parameters of U937 and HT-1080 cells using the sample rotation method [27]. Utilizing holographic optical tweezers for sample rotation, Habaza et al. have measured the 3-D RI distribution of yeast and validate measured internal structures from 3-D RI information with confocal fluorescent microscopy [35].

Measuring RI values in live eukaryotes enables to investigate cellular responses according to external environmental changes. Fang-yen et al. reported rapid increases in RI values of cytoplasm upon exposure to the acetic acid solution, while there were no significant 
changes in nucleoli [82]. In neuroscience, ODT has been used for studying neuronal connectivity using structural information of neurons (Fig. 7(c) and [23, 83]). Also, 3-D dynamics of RI distributions of lipid droplets in human hepatocytes (Huh-7) has been characterized to investigate structural and biochemical

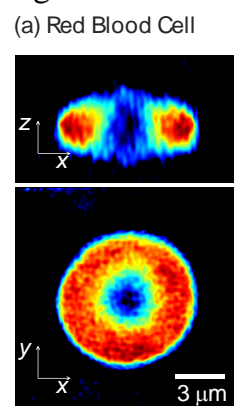

(c) Neuron Cell
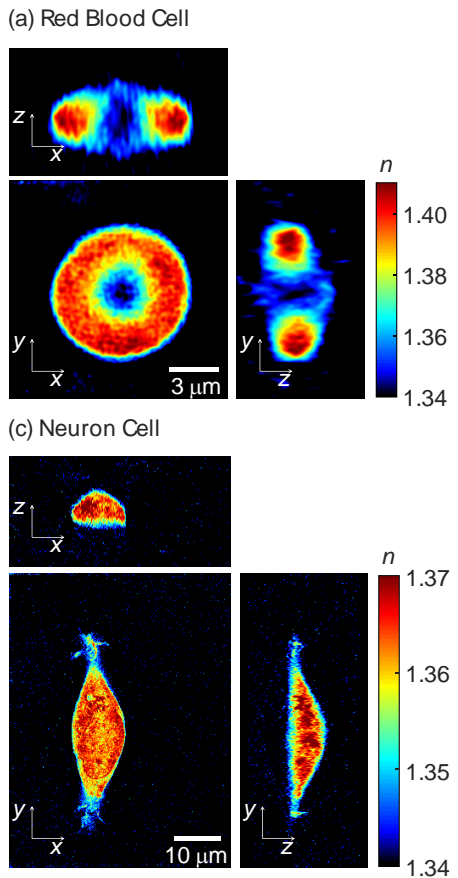

changes of internal vesicles under chemical treatment (Fig. 7(d) and [76]). 3-D shapes and internal structures of E. coli, prokaryotic cells and dynamic 3D RI images of the synaptic network were visualized using ODT with the complex deconvolution technique [23]. (b) Macrophage

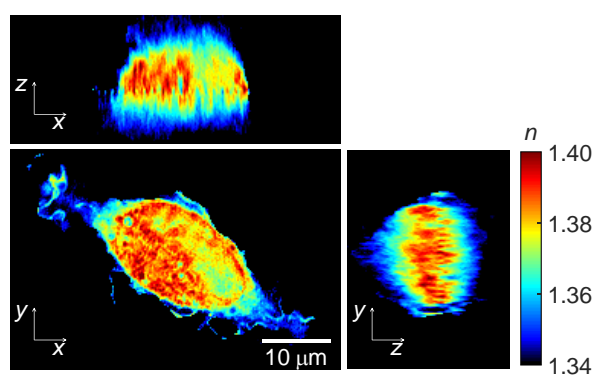

(d) Hepatocyte

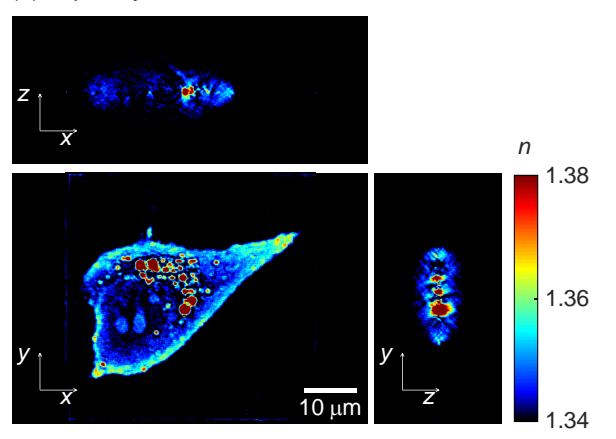

Fig. 7 Representative 3-D RI tomograms of (a) RBC, (b) macrophage, (c) neuron, and (d) hepatocyte. Color bar indicates refractive index values.

\subsection{Parasitology: malaria and babesiosis}

Malaria is an infectious disease causing significant mortality. Transmitted to humans via mosquitos, malaria-inducing parasites first invades hepatocytes, and then RBCs. Imaging and visualization techniques for the study of malaria is crucial because infectious mechanisms regarding malaria are not entirely understood [84]. ODT has shown great potential for the study of malaria because it can effectively visualize host RBCs, invaded parasites, as well as other parasiteinduced vacuole structures. Also, the label-free capability of ODT allows direct and easy visualization of malaria infected RBCs.

The 3-D RI tomograms of RBCs parasitized by Plasmodium falciparum (pf-RBCs) was measured with the optical projection algorithm by Park et al. [46]. In this work, intracellular parasites and formed hemozoin crystals were clearly measured in the reconstructed 3-D structures of pf-RBCs. Also, the decreases in $\mathrm{Hb}$ concentration in pf-RBCs due to the consumption of parasites were quantified. Recently, it was demonstrated that the diffraction algorithm provides better imaging quality for the reconstruction of pf-RBCs, in compared to the projection algorithm [38]. Reconstructed 3-D shapes of pf-RBCs in different stages of infection and hemozoin were shown in Fig. 6(a).
ODT has been successfully used for the study of babesiosis [69], an infectious disease transferring from animals to humans and showing similar to malaria, such as anemia and fever [85, 86]. Emergency human babesiosis is caused by an obligate parasite Babesia microti (B. microti), an intraerythrocytic protozoan of the genus Babesia [87, 88]. B. microti mainly invades RBCs and causes significant alteration in host RBCs.

Conventional techniques for imaging B. microtiinvaded RBCs (Bm-RBCs) are based on optical microscopy with Giemsa-staining [87, 88]. However, these only provide limited structural information: parasitized vacuoles are not labeled with Giemsa and the discrimination of parasites rely on the experience of professional technicians. Park et al. have shown that measuring 3-D RI maps offers a novel approach to the investigation of Bm-RBCs [69]. The morphological, chemical and mechanical parameters of Bm-RBCs are quantified at the individual cell level, which reveals significant changes resulting from B. microti $\neg$ parasitic invasion [Fig. 6(b)].

\subsection{Emerging applications: virus infection and cancer biology}

One of the emerging applications of ODT is the study of virus infection. Although individual viruses are too small to be optically resolved, ODT can effectively 
visualize structures of individual cells infected with the virus. Using an ODT system combined with confocal microscopy, Simon et al. have measured 3-D RI distribution and corresponding fluorescence images of human alveolar epithelial A549 cells infected with H3N2 influenza viruses (Fig. 8(a) and Ref. [89]).

Another interesting field of application is cancer biology. WC Hsu et al. have measured 3-D RI tomograms of cancerous and normal epithelial cells, and investigated backscattering light properties by applying the finite-difference-time-domain method to experimentally determined 3-D RI tomograms (Fig. 8(b) and [90]).
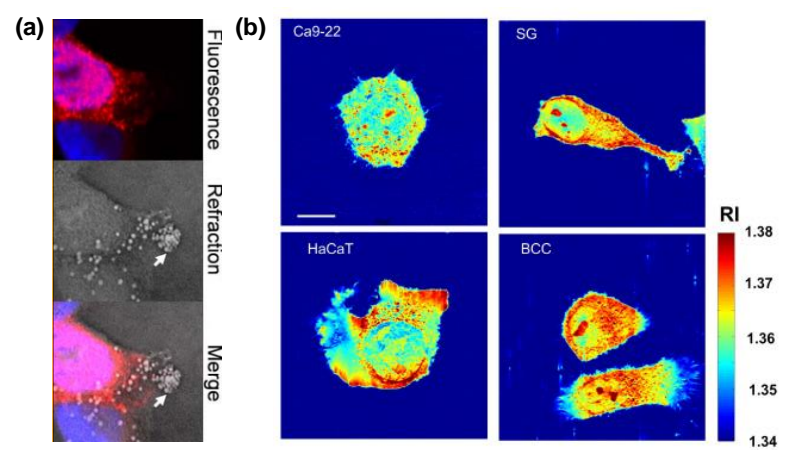

Fig. 8 3-D RI distribution of eukaryotic cells in various pathological conditions. (a) Multimodal images of human epithelial A549 cells infected with H3N2 influenza viruses. Red indicates viral nucleoprotein NP immunostaining. Blue indicates nucleus DAPI staining. Reprinted from Ref. [89] with permission. (b) $x-y$ crosssectional slices of 3-D RI tomograms of cancerous CA9-22 and BCC cell lines and two normal HaCaT and SG cell lines. Modified from Ref. [90] with permission.

\section{Concluding remarks}

In this review, the principles of ODT and their applications to the study of biological cells have been presented. The research work reviewed here and recent trends suggest that measuring 3-D RI maps of live cells may play a significant role in enhancing the understanding of cell pathophysiology. In particular, ODT will be widely used in biological and medical labs, especially when quantitative imaging capability and label-free imaging of cells are crucial such as immunotherapy using WBCs and stem cell research. However, this does not imply the ODT replaces existing optical microscopy techniques such as fluorescence confocal or multiphoton microscopy; ODT can be more efficiently used when complementary to existing cell imaging procedures.

The uses of ODT for studying cell pathophysiology have not yet been fully explored; indeed, this emerging field is at its very early stage with a few significant applications in a few fields. Considering the advantageous and potential of ODT, significant development, and wide application is expected. However, there are still many important issues in the study of cell pathophysiology using ODT that can be tackled by the good development and use of novel techniques. In particular, new techniques are needed to simplify new ODT systems. As mentioned in this review, active illumination schemes using a DMD, an SLM, or an LED array have demonstrated merits for practical applications of ODT to biological field [28, 29, 91]. The interferometric imaging techniques can also be further advanced for simple and stable measurements of optical fields. Recently reported techniques on common-path interferometric units have shown progressive advances in the interferometric imaging [24, $67,92,93]$. Also, most of previous ODT techniques have used single visible wavelengths. The use of multiple wavelengths in ODT for hyperspectral RI tomography has been recently demonstrated [77, 94], which may provide molecular specificity for some special applications [95]. More importantly, the study of cell pathophysiology using ODT will be further accelerated when biologists and medical doctors extensively utilized this technique. Very recently, ODT is commercially available by spin-off companies [96], and this will further expedite the applications of ODT.

Also, the tomographic reconstruction algorithm can be further developed. For example, current existing reconstruction algorithms are essentially based on the first order approximation and thus are not applicable to thick samples exhibiting significant multiple light scattering. To further expand the applicability of ODT from single cells to multi-layered cells, new algorithms to take into account multiple scattering should be developed, although it will be challenging due to extremely high level of computational demands. It was noteworthy that new tomographic reconstruction algorithm based on machine learning was developed [58], which will bring new insights to the problem of multiple scattering. It is noteworthy that the experimental techniques used in ODT share the same principles with the methods used in measuring transmission matrices (TM) of highly turbid media [9799]. TM relates the scattered light fields from complex media with indecent fields, which have been exploited in focusing and imaging through the complex media [100-103]. With the development of a clever reconstruction algorithm, the 3-D RI distributions of highly complex samples such as biological tissues may be directly measured with ODT.

Furthermore, regularization methods to alleviate the missing cone issues can also be advanced. Most of the conventional regularization methods are developed for the application of X-ray CT, and thus not optimized for the case of biological cells. In addition, the reconstruction speed in ODT should be enhanced for practical applications in biological and clinical settings. Recently, the use of GPU was reported to expedite the tomographic reconstruction processes in ODT [104].

Although this review article focused on the applications to the cell pathophysiology, the use of ODT can be further applied to various related research fields. For example, imaging tissue slices will provide detailed 
subcellular structures without labeling [105] or enable access to morphological features which is difficult to be imaged with conventional labeling agents. Also, as recently demonstrated, ODT can also be exploited for the study of phytoplankton [106] and hair [107] as well as industrial applications [49, 108].

We have witnessed that recent developments in optical imaging techniques have been effectively transferred from laboratories to clinics due to close interdisciplinary collaboration: successful examples include optical coherence tomography, multiphoton microscopy, and super-resolution nanoscopy. To successfully address critical issues in cell pathophysiology with ODT, it is crucial to develop interdisciplinary collaborations among clinicians, biologists, engineers and physicists. Considering the recent exponential growth of the field, we are optimistic that ODT will play important roles in various cellular studies.

\section{Acknowledgement}

This work was supported by KAIST, and the National Research Foundation of Korea (2015R1A3A2066550, 2014K1A3A1A09063027, 2012-M3C1A1-048860, 2014M3C1A3052537) and Innopolis Foundation (A2015DD126). 